\title{
Opicapone: From Preclinical Data to Real-world Evidence
}

\author{
Heinz Reichmann \\ Department of Neurology, University Hospital Dresden, Dresden, Germany
}

DOI: https://doi.org/10.17925/USN.2021.17.1.32

\begin{abstract}
$\mathrm{C}$ atechol-O-methlytransferase (COMT) inhibitors play an important role in the treatment of patients with Parkinson's disease. COMT inhibition leads to an increase in delivery of levodopa to the brain via the blood. While tolcapone inhibits COMT, both in the peripheral tissues and the central nervous system, entacapone and opicapone inhibit COMT exclusively in the peripheral system. Tolcapone has to be administered three times a day, whilst entacapone has to be added to each levodopa dose. In contrast, opicapone has to be administered only once per day, at bedtime, usually 1 hour before or after levodopa. The adverse events associated with tolcapone may include liver failure, and entacapone may cause urine discoloration or diarrhoea; however, opicapone does not cause such serious side effects. Dyskinesia, the most common side effect associated with opicapone, also occurs with the other two COMT inhibitors and can be managed by reducing the dose or the frequency of levodopa administration. There are two pivotal studies indicating that $50 \mathrm{mg}$ of opicapone provides the best balance between efficacy and tolerability compared to other doses of opicapone. These studies (BIPARK-I and -II) showed a clinically significant improvement in wearing-off symptoms and duration of symptoms in patients taking opicapone in addition to levodopa and other medication, and a gain in ON-time of about 45-60 minutes. In addition, a real-world study, OPTIPARK, substantiated these findings. Thus, clinical trials and real-world data show that opicapone is a very important drug in the quest to improve motor conditions in patients with Parkinson's disease. Additionally, some preliminary results indicate that opicapone may also improve mood, sleep and cognition.
\end{abstract}

\section{Keywords}

Parkinson's disease, levodopa, opicapone, COMT inhibition, motor fluctuations

Disclosure: Heinz Reichmann was part of the OPTIPARK study, which was sponsored by BIAL.

Review process: Double-blind peer review:

Compliance with ethics: This study involves a review of the literature and did not involve any studies with human or animal subjects performed by any of the authors.

Authorship: The named authors meet the International Committee of Medical Journal Editors (ICMJE) criteria for authorship of this manuscript, take responsibility for the integrity of the work as a whole, and have given final approval for the version to be published.

Access: This article is freely accessible at

touchNEUROLOGY.com (c) Touch Medical Media 2021.

Received: 1 March 2021

Accepted: 29 March 2021

Published online: 15 July 2021

Citation: touchREVIEWS in Neurology. 2021;17(1):32-5

Corresponding author: Heinz Reichmann, Department of Neurology, University Hospital Dresden, Fetscherstrasse 74, 01307 Dresden, Germany.E: Heinz.Reichmann@ukdd.de

Support: No funding was received for the publication of this article.
Parkinson's disease is now considered to be a spreading disease caused by the aggregation of abnormal alpha-synuclein. ${ }^{1}$ According to Braak et al., in stage I of the disease, alpha-synuclein pathology is found in the olfactory bulb and the dorsal nucleus of the vagus nerve, ${ }^{2}$ as well as in the enteric nervous system (Auerbach's and Meissner's plexuses). ${ }^{3}$ These observations fit well with the clinical features of hyposmia, constipation, depression and/or rapid eye movement (REM) sleep behaviour disorder, which characterize the so-called prodromal phase of Parkinson's disease. Parkinson's disease is a combination of motor and non-motor symptoms; the cardinal motor symptoms of akinesia, rigidity and resting tremor are caused by dopamine deficiency in the nigrostriatal pathway. For this reason, motor symptoms are predominantly treated with dopamine replacement therapy.

Guidelines, such as those of the National Institute for Health and Care Excellence, suggest that biologically young patients should first be treated with a dopamine agonist, and elderly patients, or patients in the more advanced stages of Parkinson's disease, should preferentially receive levodopa. ${ }^{4}$ Due to its high efficacy and few associated side effects, levodopa is still regarded as the gold standard. Unfortunately, levodopa, even when administered with a decarboxylase inhibitor, such as carbidopa or benserazide, has a short plasma half-life. Thus, after initial high efficacy and consequent patient satisfaction, patients experience motor fluctuations even within the first year of treatment. The first, and most abundant, motor fluctuation is wearing-off, ${ }^{5}$ caused by a reduction in the efficacy of levodopa as the disease evolves, such that each dose of levodopa is effective for a shorter time, and the symptoms of Parkinson's disease return or worsen before the next dose is due. Following the next dose of levodopa, the symptoms improve. Wearing-off is underdiagnosed, and it is mandatory to ask the patient about this motor problem. ${ }^{6}$ There are several drugs that can be used to manage wearing-off, the most frequently used of which are catechol-O-methlytransferase (COMT) inhibitors, monoamine oxidase type B inhibitors and long-acting dopamine agonists.

In this review, we will discuss the properties, preclinical data, pivotal studies and real-world data of the third-generation COMT inhibitor, opicapone. Oral opicapone is approved in the European Union, USA, Japan, Australia and other countries as adjunctive therapy to preparations of levodopa/DOPA decarboxylase inhibitors in patients with Parkinson's disease and end-of-dose motor fluctuations ${ }^{7}$ or OFF episodes. ${ }^{8}$ 


\section{Preclinical data}

So far, there are three COMT inhibitors available. Entacapone and opicapone inhibit this enzyme exclusively in the peripheral tissues, whilst tolcapone also inhibits central COMT. As tolcapone has been known to cause deleterious liver enzyme increases in some patients and fulminant liver failure in others, it can be used only as a second-line medication and with special precautions. ${ }^{9}$ The inhibition of COMT blocks the degradation of levodopa to 3-0-methyldopa (3-OMD) in peripheral tissues, resulting in more levodopa entering the blood and passing through the blood-brain barrier, increasing its bioavailability. Thus, COMT inhibitors add to the function of decarboxylase inhibitors. Decreasing the concentration of 3-OMD is also helpful because 3-OMD may compete with levodopa for passage across the blood-brain barrier.

As mentioned above, opicapone is a third-generation COMT inhibitor, following on firstly from the introduction of pyrogallol and tropolone, and then the introduction of entacapone and tolcapone. ${ }^{10}$ Similarly to entacapone, opicapone exclusively targets peripheral COMT, and there are other major differences between the two drugs. Opicapone is a hydrophilic 1,2,4-oxadiazole analogue with a pyridine $\mathrm{N}$-oxide at position 3, which leads to prolonged action (inhibition) and less cell toxicity. Entacapone is a short-acting, peripherally-selective inhibitor, which has to be administered with each dose of levodopa. Tolcapone is longer acting and more potent than either opicapone or entacapone. Kiss et al. have nicely summarized the discovery of the long-acting opicapone, ${ }^{11}$ and an excellent evaluation of the pharmacokinetics, pharmacodynamics and tolerability of opicapone in humans was undertaken by Almeida et al. ${ }^{10}$

Opicapone shows an extremely high affinity to COMT, which results in a low dissociation rate and a long duration of action in vivo, both in animals, such as monkeys, and in humans. ${ }^{12,13}$ In animal studies (rats and monkeys), opicapone proved to be safe with respect to liver metabolism, and the central nervous, cardiovascular, gastrointestinal and pulmonary systems. One hour after exposure of rats to the same dose levels of the individual inhibitors, COMT activity was inhibited by $99 \%$ with opicapone, $82 \%$ with tolcapone, and $68 \%$ with entacapone. ${ }^{13}$ Nine hours after administration, COMT was no longer inhibited by entacapone, and was only inhibited by $16 \%$ by tolcapone, whilst opicapone continued to inhibit COMT activity by $91 \%$. The finding of increased levodopa in the brain of monkeys treated with opicapone supports these findings. ${ }^{12}$

\section{Early phase clinical studies}

Similar results have been demonstrated in clinical trials in humans, where no differences were found between Asian and Caucasian healthy adult volunteers in the pharmacodynamics of $5-50 \mathrm{mg} /$ day opicapone..$^{15}$ The area under the curve for levodopa increased by $35 \%$ when $50 \mathrm{mg}$ of opicapone was added to levodopa treatment in patients with Parkinson's disease. ${ }^{16}$ Opicapone is primarily metabolized in the liver, and the resulting inactive metabolites are eliminated by excretion in the faeces and urine. Caution is only advised in patients with moderate hepatic impairment (Child-Pugh B), in whom dose adjustment is necessary. Opicapone is not recommended in patients with Child-Pugh C.

Thestudyby Almeida etal., mentioned above, was the firsttrial ofopicapone in humans, and was performed in healthy men in a single centre in Rennes, France, with 10, 25, 50, 100, 200, 400, 800 and 1,200 mg opicapone using a single-dose escalation, randomized, placebo-controlled, double-blind design. ${ }^{10}$ In a second analysis, using a single dose of $50 \mathrm{mg}$ opicapone, interaction with food was tested in a fasting and non-fasting situation. ${ }^{10}$ opicapone was well tolerated at all of the doses tested. The maximum plasma concentration was equivalent to the administered opicapone doses. The half-life of opicapone-induced COMT inhibition in human erythrocytes from these healthy volunteers was 61.6 hours and was independent of the dose. Rocha et al. also assessed the tolerability, pharmacokinetics and inhibitory effect of opicapone in healthy male subjects following repeated doses of opicapone. ${ }^{16}$ Their study reaffirmed the previous finding that the inhibition of COMT by opicapone is of long duration, and showed that once-daily dosing of opicapone is adequate, which may provide an advantage over entacapone and tolcapone. ${ }^{16}$

\section{Pivotal trials}

There are two phase III, double-blind, multicentre, multinational trials - BIPARK- $1^{17}$ and BIPARK-II18 - in patients with Parkinson's disease with end-of-dose motor fluctuations. Both were short-term trials with an observation period of 14-15 weeks. BIPARK-I also evaluated the non-inferiority of opicapone to entacapone.

In BIPARK-I, 600 patients were randomly assigned to opicapone, entacapone or placebo. The study was performed in 106 specialized centres in Europe and Russia. ${ }^{17}$ The primary endpoint was the change from baseline to end of study treatment in absolute time in the OFF state, as assessed by daily paper patient diaries. A total of 120 patients receiving placebo, 120 receiving $200 \mathrm{mg}$ entacapone with each levodopa dose, 119 receiving $5 \mathrm{mg}$ opicapone once daily, 116 receiving $25 \mathrm{mg}$ opicapone once daily and 115 patients receiving 50 mg opicapone once daily, in addition to levodopa, were included in the full analysis set, and 537 patients were included in the per-protocol set. The mean change in time in the OFF state was -56.0 minutes for placebo, -96.3 minutes for entacapone, -91.3 minutes for $5 \mathrm{mg}$ opicapone, -85.9 minutes for $25 \mathrm{mg}$ opicapone and -116.8 minutes for $50 \mathrm{mg}$ opicapone. Both a significant superiority to placebo and non-inferiority to entacapone were demonstrated when using $50 \mathrm{mg}$ opicapone. Treatment-emergent adverse events were reported in $50 \%$ of placebo-treated patients, $57 \%$ of the entacapone group, $52 \%$ of the $5 \mathrm{mg}$ opicapone group, $55 \%$ of the 25 mg opicapone group and $54 \%$ of the $50 \mathrm{mg}$ opicapone group. The most common adverse event was dyskinesia (18 out of 115 patients treated with $50 \mathrm{mg}$ opicapone). The authors conclude that the addition of $50 \mathrm{mg}$ opicapone to levodopa treatment in patients with Parkinson's disease and end-of-dose fluctuations could enable a simplified drug regimen that allows individual tailoring of the existing levodopa daily regimen. The authors postulate that the addition of opicapone in these patients has the potential to reduce the daily levodopa dose and extend the dosing interval, thus reducing the number of doses and perhaps increasing adherence.

In BIPARK-II, Lees et al. evaluated the efficacy and safety of 25 and $50 \mathrm{mg} /$ day dosages of opicapone as adjunct to levodopa therapy in patients with Parkinson's disease experiencing end-of-dose motor fluctuations, in a phase III, international, multicentre, outpatient, double-blind, randomized, placebo-controlled study. ${ }^{18}$ As in BIPARK-I, the observation period was 14-15 weeks, and patients with end-of-dose fluctuations and total awake OFF-time (excluding early morning akinesia) of at least 1.5 hours received 25 or $50 \mathrm{mg} /$ day of opicapone, followed by a 1-year open-label phase, during which all patients received active treatment with opicapone $25 \mathrm{mg}$ or 50mg. The primary efficacy outcome of the double-blind phase was the change from baseline in absolute OFF-time versus placebo, based on patient diaries. The open-label phase focused on maintenance of treatment effect in OFF-time.

A total of 427 patients (258 men and 169 women with a mean age of $63.1 \pm 8.8$ years) were randomized to a $25 \mathrm{mg} /$ day $(n=129)$ or $50 \mathrm{mg} /$ day $(n=154)$ dosage of opicapone or to placebo $(n=144)$. 
Of these, 367 patients completed the double-blind phase and entered the open-label phase. Two hundred and eighty-six patients (78\%) completed 1 year of open-label treatment. At the end of the double-blind phase, the least squares mean change (standard error [SE]) in OFF-time was -64.5 (14.4) minutes for the placebo group, -101.7 (14.9) minutes for the $25 \mathrm{mg} /$ day opicapone group, and -118.8 (13.8) minutes for the $50 \mathrm{mg} /$ day opicapone group. Only opicapone treatment in the $50 \mathrm{mg}$ group was statistically significantly more efficacious than placebo. The OFF-time reduction in the $50 \mathrm{mg}$ group was sustained throughout the open-label phase (-126.3 minutes at 1-year open-label endpoint). The most common adverse events in the opicapone versus placebo groups were dyskinesia, constipation and dry mouth. Fifty-one patients (14\%) were discontinued from the study during the double-blind phase. The efficacy of $50 \mathrm{mg}$ opicapone was similar in both the BIPARK-I and BIPARK-II studies, and both studies showed that some patients might develop dyskinesia after the addition of opicapone; this can be controlled by decreasing the total levodopa dose.

Ferreira et al. analysed the efficacy of opicapone (25 and $50 \mathrm{mg}$ ) as adjunct therapy to levodopa in a pooled population from BIPARK-I and BIPARK-II. In addition, they evaluated the outcome in all patients who underwent the 1-year open-label extensions of both BIPARK-I and BIPARK-II. ${ }^{19}$ Double-blind treatment with opicapone (25 and $50 \mathrm{mg}$ ) significantly reduced absolute daily OFF-time from a baseline of 6.1-6.6 hours. The mean change in OFF-time versus placebo was -35.1 minutes $(p=0.0106)$ for the $25 \mathrm{mg}$ dose and -58.1 minutes $(p<0.0001)$ for the $50 \mathrm{mg}$ dose. Accordingly, significant increases in ON-time without troublesome dyskinesia were recorded $(p<0.05$ and $p<0.0001$ for the 25 and $50 \mathrm{mg}$ doses, respectively). Interestingly, no significant differences were observed for ON-time with troublesome dyskinesia between placebo and opicapone. Patients recorded their mobility using diaries during the open-label phase. There was a maintenance of effect for patients previously treated with opicapone $50 \mathrm{mg}$. The group previously treated with the $25 \mathrm{mg}$ dose benefitted with further optimization of therapy during the open-label phase, whilst switching from placebo to opicapone led to significant reductions in OFF-time and increased ON-time. This study demonstrated a persistent efficacy of opicapone during the observation period of about 16 months with a reduction in OFF-time and gain in ON-time without troublesome dyskinesia.

In a more recent work, Ferreira et al. described patients who had initially been treated with entacapone in the double-blind trial BIPARK-I, then were switched to opicapone and followed in an open-label phase for 12 months. ${ }^{20}$ This cohort improved with a further 39.3 minutes reduction of OFF-time from baseline. Thus, this post hoc analysis supports the conclusion that opicapone may be more efficacious than entacapone in reducing OFF-time.

\section{Real-world data}

The open-label OPTIPARK trial on the efficacy and safety of opicapone was recently performed in the UK and Germany. ${ }^{21}$ This was a prospective, open-label, single-arm study that included patients with Parkinson's disease with motor fluctuations. The observation period was 3 months in Germany and 6 months in the UK. The primary endpoint was the change in Clinician's Global Impression of Change scale (CGI-C) after treatment with $50 \mathrm{mg} /$ day opicapone in clinical practice. Secondary endpoints included safety and efficacy, and changes in Patient's Global Impression of Change scale (PGI-C), Unified Parkinson Disease Rating Scale (UPDRS), quality of life (Parkinson's disease Questionnaire-8) and non-motor symptoms (Non-Motor Symptoms scale [NMSS]). Male and female patients ( 230 years of age) with idiopathic Parkinson's disease (based upon the UK Parkinson's Disease Society Brain Bank Clinical Diagnostic Criteria), and with end-of-dose akinesia/wearing-off (using The Wearing-off Questionnaire) were included in the study. Additional inclusion criteria included Hoehn and Yahr stages I-IV (during ON periods), treatment with 3-7 daily doses of levodopa/decarboxylase inhibitor or triple therapy with entacapone, and the clinical impression of the treating neurologist. Exclusion criteria were atypical Parkinson's disease, unpredictable OFF periods and severe hepatic impairment (Child-Pugh C).

Five hundred and six patients were enrolled in 68 centres across Germany and the UK. There were four screening failures, 495 patients received at least one dose of opicapone and 393 patients completed the first 3 months; 109 patients prematurely terminated treatment (non-serious adverse events $n=76$; withdrew consent $n=14$; serious adverse events $n=8$; lack of efficacy $n=3$; development of exclusion criterion $n=2$; and other $n=4$ ). The 495 patients had a mean age of 67.7 years, two-thirds were men, all were Caucasian, the mean duration of Parkinson's disease was 102 months and the mean duration of motor fluctuations was 30 months. Total daily mean levodopa dose was $580 \mathrm{mg}$ for all patients, with additional rasagiline in $28 \%$, pramipexole in $25 \%$, ropinirole in $22 \%$, amantadine in $21 \%$, rotigotine in $14 \%$, safinamide in $14 \%$ and piribedil in $9 \%{ }^{21}$

The main outcome of this open-label study was that after 3 months of treatment with opicapone, clinical improvement was judged to have occurred in $71.3 \%$ of the patients by GCl-C scale and $76.9 \%$ of the patients by PGI-C scale, overall. After 6 months, the UK sub-cohort $(n=95)$ had improved imrpoved further ( $85.3 \%$ by GCl-C scale). UPDRS Part II (activities of daily living during OFF period) scores improved by $3.0 \pm 4.6$ points $(p<0.0001)$ after 3 months of treatment. Quality of life and NMSS also significantly improved (change in NMSS $-6.8 \pm 19.7$ points). Amongst non-motor symptoms, which improved with the addition of opicapone, sleep/fatigue and mood/cognition were the most responsive. After the 3-month treatment period, most patients had unchanged levodopa doses and dosing frequency. ${ }^{21}$

Side effects, mostly considered mild or moderate, occurred in $74.9 \%$ of patients. Thirty-four (7.1\%) patients developed serious side effects. The most frequent treatment-related side effects were dyskinesia (11.5\%), dry mouth (6.5\%) and dizziness (4.8\%). Since diarrhoea is a common side effect of entacapone, it is interesting to note that only $0.6 \%$ of patients in the OPTIPARK trial developed diarrhoea.

In summary, OPTIPARK presented real-world clinical data on the efficacy and safety of $50 \mathrm{mg}$ opicapone. The results were very similar to the two pivotal studies discussed above. After 3 months, opicapone caused significant improvements, not only in UPDRS and CGI-C/ PGI-C scores, but also in quality of life and non-motor symptoms. Side effects were in the same range as in the pivotal studies, with dyskinesia being the most frequent one. $\square$
1. Olanow CW, Brundin P. Parkinson's disease and alphasynuclein: is Parkinson's a prion-like disorder? Mov Disord. 2013;28:31-40.

2. Braak H, de Tredici K, Rüb U, et al. Staging of brain pathology related to sporadic Parkinson's disease. Neurobiol Aging. 2003;24:197-211.
3. Braak H, de Vos RA, Bihl J, del Tredici K. Gastric alpha-synuclein immunoreactive inclusions in Meissner's and Auerbach's plexuses in cases staged for Parkinson's disease-related brain pathology. Neurosci Lett. 2006;396:67-72.

4. National Institute for Health and Care Excellence. Parkinson's disease in adults. NICE guideline [NG71]. London: NICE, 2017.
Available at: www.nice.org.uk/guidance/NG71 (accessed 10 June 2021).

5. Stacy M. The wearing-off phenomenon and the use of questionnaires to facilitate its recognition in Parkinson's disease. J Neural Transm. 2010;117:837-46.

6. Stocchi F, Antonini A, Barone $P$, et al. Early DEtection of WEaring 
off in Parkinson disease; the DEEP study. Parkinsonism Relat Disord. 2014;20:204-11

7. Electronic Medicines Compendium. Ongentys $50 \mathrm{mg}$ hard capsules. Summary of product characteristics. Available at: www.medicines.org uk/emc/product/7386\#gref Available at: WwW.medicines
(accessed 19 April 2021)

8. US Food and Drug Administration. Ongentys Highlights of Prescribing information. Available at: www.accessdata.fda.gov/drugsatfda_docs/ label/2020/212489s000lbl.pdf (accessed 19 April 2021)

9. $\operatorname{Tasmar}^{\circledast}$ (tolcapone) Prescribing Information. Available at: www.accessdata.fda.gov/drugsatfda_docs/ label/2013/020697s004lbl.pdf (accessed 14 July 2021).

10. Almeida L, Rocha JF, Falcao A, et al. Pharmacokinetics, pharmacodynamics and tolerability of opicapone, a novel catechol-o-methyltransferase inhibitor, in healthy subjects. Clin Pharmacokinet. 2013;52:139-51.

11. Kiss $L E$, Ferreira $H S$, Torrao $L$, et al. Discovery of a long-acting, peripherally selective inhibitor of catechol-O-methyltransferase. peripherally selective inhibitor of cat
J Med Chem. 2010;53:3396-411.
12. Bonifacio MJ, Sutcliffe JS, Torrao L, et al. Brain and peripheral levodopa pharmacokinetics in the cynomolgus monkey following administration of opicapone, a novel catecholO-methyltransferase inhibitor. Parkinsonism Relat Disord. 2012;18(Suppl. 2):S125.

13. Bonifacio MJ, Torrao L, Loureiro AL, et al. Opicapone: characterization of a novel peripheral long-acting catecholO-methyltransferase inhibitor. Parkinsonism Relat Disord. 2012;18(Suppl 2):S125.

14. Falcao A, Rocha JF, Santos A, et al. Opicapone pharmacokinetics and pharmacodynamics comparison between healthy Japanese and matched white subjects. Clin Pharmacol Drug Dev. 2016;5:150-61.

15. Rocha JF, Ferreira JJ, Falcao A, et al. Effect of 3 single-dose regimens of opicapone on levodopa pharmacokinetics, catechol0 -methyltransferase activity and motor respone in patients with Parkinson disease. Clin Pharmacol Drug Dev. 2015;5:232-40. 16. Rocha JF, Almeida L, Falcao A, et al. Opicapone: a short lived following multiple dose administration in healthy subjects.
Br J Clin Pharmacol. 2013;76:763-75

17. Ferreira JJ, Lees A, Rocha JF, et al. Opicapone as an adjunct to levodopa in patients with Parkinson's disease and end-of-dose motor fluctuations: a randomised, double-blind, controlled trial. Lancet Neurol. 2016;15:154-65.

18. Lees AJ, Ferreira J, Rascol O, et al. Opicapone as adjunct to levodopa therapy in patients with Parkinson disease and motor fluctuations: a randomized clinical trial. JAMA Neurol. 2017; 74:197-206

19. Ferreira JJ, Lees A, Rocha JF, et al. Long-term efficacy of opicapone in fluctuating Parkinson's disease patients: a pooled analysis of data from two phase 3 clinical trials and their openlabel extensions. Eur J Neurol. 2019;26:953-60.

20. Ferreira JJ, Lees A, Poewe W, et al. Effectiveness of opicapone and switching from entacapone in fluctuating Parkinson disease. Neurology. 2018;90:e1849-57.

21. Reichmann H, Lees A, Rocha JF, et al. Effectiveness and safety of opicapone in Parkinson's disease patients with motor fluctuations: the OPTIPARK open-label study. Translat Neurodegen . 2020:9:9. 